\title{
Lexis
}

Journal in English Lexicology

$8 \mid 2014$

Metaphor Studies in the English Language

\section{Métaphore grammaticale : le nom en -ness, une création lexicale à usage unique}

Julie Neveux

\section{CpenEdition}

Journals

Édition électronique

URL : http://journals.openedition.org/lexis/261

DOI : 10.4000/lexis.261

ISSN : 1951-6215

Éditeur

Université Jean Moulin - Lyon 3

Référence électronique

Julie Neveux, « Métaphore grammaticale : le nom en -ness, une création lexicale à usage unique », Lexis [En ligne], 8 | 2014, mis en ligne le 09 janvier 2014, consulté le 19 avril 2019. URL : http:// journals.openedition.org/lexis/261; DOI : 10.4000/lexis.261

Ce document a été généré automatiquement le 19 avril 2019

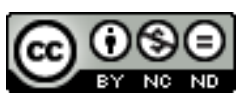

Lexis is licensed under a Creative Commons Attribution-NonCommercial-NoDerivatives 4.0 International License. 


\title{
Métaphore grammaticale : le nom en -ness, une création lexicale à usage unique
}

\author{
Julie Neveux
}

\section{Introduction : créativité}

1 À l'entrée -ness, l'Oxford English Dictionary (OED) donne les quelques exemples suivants, correspondant tous, précise le dictionnaire, à des emplois distinctifs et uniques du suffixe depuis le XIX ${ }^{\mathrm{e}}$ siècle :

1804 S. T. Coleridge Let. in Lit. Remains (1836) II. 414 The exclusive SirThomas-Brown-ness of all the fancies.

1853 'G. Eliot' in J. W. Cross George Eliot's Life (1885) I. 319 Dislike-to-gettingup-in-the-morningness.

1859 G. A. Sala Gaslight \& Daylight iv. 43 An irreproachable state of cleanshirtedness, navy blue-broadclothedness and chimney-pot-hattedness.

1901 Academy 8 June 495/2 Southport, with its sponge-cakeyness and schoolgirlism is surely worth study.

1949 P. Grainger Let. 23 Nov. in All-round Man (1994) 240 You are a lovechild moving towards art. I am an artist moving towards love-child-ness.

2000 Guardian 24 Mar. (Review section) 21/1 The numbskulled singalongness of Oasis.

2 Écrivains, poète ou journalistes, c'est le métier des auteurs de ces nominalisations en ness que de savoir manier le langage, et son potentiel expressif. Ce sont tous, dans une certaine mesure, des (re)créateurs de discours. Dans ces noms complexes en -ness, le suffixe s'est attaché à des bases diverses, nominales ou adjectivales, déjà composées pour la plupart (nom composé - love-child, adjectif composé - clean-shirted, etc.). La nominalisation de Coleridge (citation 1) porte sur un nom propre affecté de son titre, Sir Thomas Brown. Si « inventives » sont ces créations lexicales qu'il semble logique que leur 
emploi soit unique. Ces noms en -ness sont des noms ad hoc, des noms créés dans un contexte particulier, avec une motivation particulière - il est impossible de les réutiliser. Pourtant, c'est l'objet de cet article, ces 6 cas de nominalisation sont des cas prototypiques de nominalisation en -ness, c'est-à-dire qu'ils réunissent le plus grand nombre possible de caractéristiques spécifiques à ce processus de dérivation lexicale. Parce que les auteurs de ces 6 énoncés ont fait preuve d'une créativité perceptible à un haut degré à la surface du discours, la motivation de la nominalisation, qui est de nature métaphorique, est perceptible à un haut degré également. Dans les énoncés de la vie quotidienne, dont la métaphoricité est moins saillante, c'est toujours le même besoin créatif qui préside aux nominalisations en -ness, comme le montre la conversation suivante, donnée par le COCA (Corpus of Contemporary American English). Nous sommes dans une émission de télévision culinaire. Le chef, Frank Bonanno, décrit les étapes de sa recette :

Mr-Bonanno: Then you put this over the heat. RodRiguez: OK. Mr-Bonanno: Cook it and... RODRIGUEZ: How long do you cook this, Frank? Mr-BONANNO: This would take about probably eight minutes, six to eight minutes. RODRIGUEZ: OK. Quick, very quick. Mr-BonanNo: Yeah. And as it melts down, the sugars will melt and the fruit will release some of the residual water in it. RODRIGUEZ: Mm. All right. Let's take a look at what it looks like when it's all done. Look at that beautifulness. Is that a word? JEFF-GLOR-co-host: That's a word'.

La nominalisation porte ici sur la base adjectivale beautiful. Bien qu'elle soit en apparence plus banale, puisque déjà utilisée - le nom beautifulness est une entrée de l'OED, ce qui atteste la lexicalisation de la nominalisation en question, le sujet parlant (Rodriguez) a eu le sentiment (besoin), en la réalisant, de créer un nouveau mot, comme le prouve sa question dans le co-texte immédiat de droite: Is that a word? "C'est bien un mot », le rassure son interlocuteur. Non seulement c'est un mot, mais c'est une métaphore grammaticale, discrète, qui répond à une situation énonciative typique (de nature phénoménologique) et a vocation, au moment précis où elle est prononcée, à un emploi unique. Il importe de remarquer en effet que c'est souvent par différence avec un mot existant qu'un nom en -ness est proposé. La nominalisation en -ness enregistre un rejet momentané, conscient ou non, du lexique disponible. Beauty existe, mais beautifulness lui est préféré. Qu'apporte le suffixe -ness, que parvient-il à transmettre de plus que le nom abstrait beauty?

Dans l'optique cognitiviste des travaux de Langacker [1990, 2008, 2009], Cuickens et alii [2003], Panther et alii [2009], j'interprète la nominalisation en -ness comme une métaphore grammaticale, dont la perceptibilité (saillance) diminue en même temps qu'elle se lexicalise. Afin d'argumenter cette position, je compare les fonctionnements de -ness et de son suffixe "rival», -ity, dans un corpus du XVII ${ }^{e}$ siècle ${ }^{2}$, les Devotions Upon Emergent Occasions de John Donne, méditations métaphysiques en forme de journal « intime » adressé à Dieu, que le prédicateur anglican rédige à la fin de l'année 1623, lorsqu'il se remet tout juste d'une maladie violente et difficile à interpréter. 


\section{1. -ness et -ity}

\subsection{L'inflation de l'emploi de -ness}

5 américains, d'une certaine inflation dans l'usage du suffixe -ness; un article du Times daté du 2 mai 1962 s'en inquiète en ces termes :

Now comes an equally formidable enemy: -ness (equally as much a "barbarism" as businesswise, dollarwise, salewise, and weatherwise] denoting "state, quality, or condition." It is not the friendly suffix of greatness, goodness, loveliness (properly forming abstract nouns from adjectives) or even LochNess, but a whole new invasion of language... Teacher Foote reports that ness added to nouns, pronouns, verbs, and phrases - a custom thought until now to be mostly whimsical, as in whyness, and everydayness - has become popular among distinctly unjocose people... Teacher Foote has spotted the malpractice as far back as a rare 16th century book that describes Fingal's Cave in the Hebrides as having cavernnesse.

6 Tout en se moquant de cette réaction « sentimentale » à un fait de langue (le suffixe est-il plus ou moins «sympathique», friendly?), Williams [1965: 285-286] confirme la productivité de-ness, et ce, aux dépens du suffixe-ity:

Another point to be made is that the -ness suffix seems to be thriving at the expense of the -ity suffix. Of the 136 words listed above ${ }^{4}$, approximately 46 have noun equivalents, in widespread use, that end in -ity; but among my students, purity, humanity, humility, and others are now almost always replaced by pureness, humanness, and humbleness, with no connotation conveyed by the suffix, though there can be little doubt that the -ity words are a part of the students' reading vocabulary.

Les deux suffixes -ness et -ity s'ajoutent à des adjectifs pour former des noms ; cependant, si certaines bases acceptent les deux suffixes, comme porous, qui fait porousness et porosity, d'autres, pour des raisons mal déterminées selon The Cambridge Grammar of the English Language, n'acceptent que l'un des deux. Par exemple, linear préfère -ity, et bearded -ness. Mais, même lorsque les bases sont compatibles avec les deux suffixes, -ness l'emporte le plus souvent.

\subsection{Productivité et transparence}

Selon Bauer [2001 : 41], la productivité est une question de potentialité créatrice :

productivity is all about potential. A process is productive if it has the potential to lead to new coinages, or to the extent to which it does lead to new coinages. We are aware of productivity only through the new coinages and the patterns of familiar and unfamiliar words coined by the relevant process.

9

Parmi les raisons de la plus grande productivité de -ness, Williams [1965 : 286] avance les suivantes :

There are several reasons for the widespread use of -ness, aside from its common use with simple adjectives. One reason is that, compared with the -ity suffix, it is shorter, adding only one syllable to the base while -ity adds two; a second reason is that it does not necessitate a phonetic shift, as in futile-futility, for example. But even more important than this is what Jespersen called 'value stressing'-here the desire to keep the full force of the adjective as the most important item to be 
communicated. This is what accounts for the preference for miserableness and maliciousness rather than for the shorter forms, misery and malice. ness: absence de changement vocalique, de déplacement de l'accent, et absence de modification de la syllabation de la base. Le suffixe -ness est en effet un suffixe «transparent », si l'on appelle «transparent » avec Bauer [2001] le nom suffixé dont la base n'est pas modifiée : "the phonology of the base word (including stress) is preserved". Or la transparence est un facteur de productivité, productivité définie par le potentiel de formation de mots davantage que par leur lexicalisation - leur entrée dans un dictionnaire. La lexicalisation n'est pas un trait définitoire de la productivité, selon Bauer [2001: 97-98] : "productivity is a feature of morphological innovation. It is a feature of morphological processes which allow for new coinages, but not all coining necessarily". Au contraire, la lexicalisation est le point d'aboutissement - figement catégorisant - d'un processus créatif caractérisé par une motivation décatégorisante. Bauer [2001: 160] confirme que le suffixe ness compte parmi les trois plus productifs de la langue anglaise avec -able et -er, et insiste [1983 : 222, on souligne] sur sa simplicité :

-ness is one of the most productive suffixes in the English language today. It is added predominantly, though not exclusively, to adjectives, and is used in nonce formations, and even established words, to replace other suffixes producing nouns from adjectives. [...] semantically it is one of the simplest of these suffixes, and consequently it often gives rise to pairs of words which some speakers distinguish semantically, e.g. sincereness/sincerity, productiveness/productivity.

Marchand [1960: 272] parle d'une " compétition » entre les suffixe -ity et -ness: The sbs (substantives) often compete and we have sense of other variants such as accuracy/ accurateness, entireness/entirety. Or cette compétition doit être interprétée selon Bauer [1983 : 82], Riddle [1985] et Baeskow [2012]. Les suffixes -ity et -ness ne sont pas interchangeables. Lorsque le sujet parlant préfère « inventer » un nom en -ness lorsque le nom en -ity préexiste, c'est un fait de langue signifiant.

\subsection{Une dérivation perceptible}

La transparence induite par le suffixe -ness joue un rôle dans la productivité du suffixe ; ity est moins transparent, induisant changement vocalique et de syllabation. Avec la suffixation en -ity, le déplacement d'accent affecte la qualité de la voyelle, comme dans clear qui donne clarity ou clearness. Ce que permet cette transparence, c'est bien la conservation totale de l'origine prédicative du nom; dans le nom en -ness, l'adjectif continue à s'exprimer avec force. Mais l'effet produit par la transparence du suffixe est interprété différemment selon les linguistes : Zajicek [1965:46, il souligne] remarque que la terminaison saxonne -ness indique la qualité ou l'état, mais « de façon plus directe que les suffixes romans", puisque "le maintien d'une racine inchangée accuse encore le caractère plus immédiat, presque sensoriel, du suffixé en ness ». « Immédiat », « direct », « sensoriel »: la transparence est en réalité l'indice d'une créativité énonciative, car elle laisse s'exprimer, ensemble, les deux catégories de discours, et fait percevoir à l'interlocuteur l'engagement du sujet parlant dans son discours. Le propre du suffixe -ness est de permettre une décatégorisation qui soit audible à l'oral, visible à l'écrit, perceptible en tout cas, créant ainsi une signification qui excède le discours pour renvoyer aux paramètres énonciatifs dans ce qu'ils ont de particulier, d'unique. Convenir à une production discursive différentielle requiert un trait spécifique - de la souplesse. 
ne autre raison essentielle de la productivité exceptionnelle de -ness réside dans la souplesse de son emploi, visible dans les exemples donnés par l'OED cités plus haut : -ness se suffixe à n'importe quelle catégorie grammaticale à l'exception du verbe, et ce, bien que le phénomène se soit amplifié, ou soit devenu plus visible à la fin du $\mathrm{xx}^{\mathrm{e}}$, depuis au moins le Moyen Anglais. Le suffixe est en effet d'origine germanique, existe depuis le Vieil Anglais, et, selon l'OED, sert la plupart du temps à exprimer une condition, un état ou une qualité par un processus d'«abstraction». Marchand [1960: 271] énumère ainsi ses propriétés principales:

Forms abstract sbs (substantives) with the meaning 'state, condition, quality of. Since OE it has chiefly been tacked on to adjs and participal adjs, but in ModE it is also used with various other bases, but not with verbs.

Exs of deadjectival derivatives are bitterness, brightness, cleanness, coolness, darkness, deepness, dimness, drunkenness, evenness, fairness, fatness, fleshliness, goodness, greediness, greenness, grimness, [...] all recorded in the OE period. The sf has been appended to adjs of native as well as foreign origin. From the ME period or later are recorded such words as absoluteness, acuteness, alimentiveness, ancientness, aptness, archness [...], readiness, sickness, straitness (etc.).

Marchand [1960: 159] note donc la productivité précoce des suffixes « natifs » à partir de bases anglaises ou empruntées :

Suffixes such as -ful, -less, -ness were early used with French words so we find faithful, faithless, clearness and others recorded by 1300 .

Alors que le suffixe -ity, d'origine latine, fait preuve d'une moins grande souplesse, des suffixations en -ness à partir de bases multiples ont été faites à toutes les époques de l'évolution de la langue anglaise. L'OED met aussi en avant la souplesse de cette dérivation, puisque le suffixe peut prendre pour base des mots composés ou des syntagmes adjectivaux comme get-at-ableness, des pronoms, Iness, meness, et même des syntagmes nominaux déjà complexes, comme l'exemple pris dans George Eliot's Life ${ }^{5}$, dislike-to-getting-up-in-the-morningness. -ness peut ainsi s'ajouter à premier suffixe flexionnel de participe présent ou passé alors qu'aucun autre suffixe dérivationnel ne le fait.

Le suffixe -ness est ainsi plus propice que -ity à l'expression d'une créativité lexicale stimulée par une expérience particulière. Vérifions à présent en contexte cette différence, en définissant le type de situation énonciative présidant à l'émergence d'une nominalisation en -ness.

\section{4. Étude en contexte : infinity/infiniteness}

Dans le texte de Donne, les noms infinity et infiniteness apparaissent dans les contextes suivants :

No man is so little, in respect of the greatest man, as the greatest in respect of God; for here, in that, we have not so much as a measure to try it by; proportion is no measure for infinity. He that hath no more of this world but a grave; he that hath his grave but lent him till a better man or another man must be buried in the same grave; he that hath no grave but a dunghill, he that hath no more earth but that which he carries, but that which he is, he that hath not that earth which he is, but even in that is another's slave, hath as much proportion to God, as if all David's worthies, and all the world's monarchs, and all imagination's giants, were kneaded and incorporated into one, and as though that one were the survivor of all the sons of men, to whom God had given the world [Exp. 2: $7^{6}$ ] 
Lay hold upon his soul, O God, till that soul have thoroughly considered his account; and how few minutes soever it have to remain in that body, let the power of thy Spirit recompense the shortness of time, and perfect his account before he pass away; present his sins so to him, as that he may know what thou forgivest, and not doubt of thy forgiveness, let him stop upon the infiniteness of those sins, but dwell upon the infiniteness of thy mercy; let him discern his own demerits, but wrap himself up in the merits of thy Son Christ Jesus; breathe inward comforts to his heart, and afford him the power of giving such outward testimonies thereof, as all that are about him may derive comforts from thence, and have this edification, even in this dissolution, that though the body be going the way of all flesh, yet that soul is going the way of all saints [Pr.17: 102].

That that blessed Son of thine may have the consummation of his dignity, by entering into his last office, the office of a judge, and may have society of human bodies in heaven, as well as he hath had ever of souls; and that as thou hatest sin itself, thy hate to sin may be expressed in the abolishing of all instruments of so, the allurements of this world, and the world itself; and all the temporary revenges of sin, the stings of sickness and of death; and all the castles, and prisons, and monuments of sin, in the grave. That time may be swallowed up in eternity, and hope swallowed in possession, and ends swallowed in infiniteness, and all men ordained to salvation in body and soul be one entire and everlasting sacrifice to thee, where thou mayst receive delight from them, and they glory from thee, for evermore [Pr. 18: 110].

Les syntagmes nominaux incluant les noms suffixés sont donc:

- $\varnothing$ infinity

- the infiniteness of those sins

- the infiniteness of thy forgiveness

- $\varnothing$ infiniteness

Dans aucun des trois extraits n'apparaît, dans un contexte proche, la base adjectivale infinite. Le nom suffixé infinity de la citation 1 est inclus dans un propos général à portée universelle, il fait partie d'un énoncé à valeur générique : "proportion is no measure for infinity". Il s'agit ici de la notion abstraite d'infini, à laquelle il est fait référence à l'aune de la notion de proportion, la première, spécifiquement divine, ne permettant qu'on lui compare la seconde, spécifiquement humaine. C'est donc un cas typique d'emploi de notion abstraite, avec le déterminant zéro. La qualité infinite n'est considérée que par rapport à elle-même, détachée de tout support.

Les emplois de infiniteness, au contraire, ancrent la qualité dans son support. Dans la citation 2, la prédication originelle est explicite dans la structure de ces syntagmes, avec, en complément du nom, les syntagmes those sins et thy mercy, sujets dans la genèse de la construction de l'énoncé. Ainsi on reconstruit aisément les premiers énoncés implicites :

\begin{tabular}{|l|l|l|}
\hline Those sins are infinite & $>$ & the infiniteness of those sins \\
\hline Thy mercy is infinite & $>$ & the infiniteness of thy mercy \\
\hline
\end{tabular}

21 La nominalisation ne masque pas la prédication première. La suffixation en -ness conserve ici les traces de l'origine prédicative malgré la dérivation nominale. Si les travaux de Langacker et d'autres grammairiens cognitivistes révèlent la forte identité de la partie nominale du discours, Goes suggère que l'adjectif possède lui aussi une identité catégorielle, longtemps méconnue, qu'un passage vers une autre partie du discours ne lui 
fait pas perdre: Goes [1999: 129] insiste sur l'« extraordinaire souplesse» de cette catégorie, ainsi que sur son mécanisme de référence syncatégorématique, à « incidence externe » dans les termes guillaumiens, concluant [1999: 227] que « l'adjectif a bel et bien une identité morphosyntaxique et sémantique propre». Goes [1999: 278] vérifie le caractère opératoire des quatre critères classiques de définition de l'adjectif : «l'accord en genre et en nombre, la gradation par très, la fonction épithète, la fonction attribut ». Ce n'est qu'en combinant ces critères que l'on obtient un prototype adjectival abstrait, et que l'on peut déterminer le degré d'« adjectivité » d'un adjectif, ou d'une autre catégorie de discours.

Dans le cas des syntagmes the infiniteness of those sins et the infiniteness of thy mercy, les supports de la prédication sont explicitement mentionnés, comme si le nom hésitait entre les catégories nominale et adjectivale et gardait le souvenir de son fonctionnement syncatégorématique. La fonction « attribut » persiste dans la catégorie du nom, signalant un certain degré d'adjectivité du nom en -ness. Si l'on revient à la définition logique de l'abstraction, on voit qu'infiniteness, dans ces syntagmes, n'a d'«abstrait " que son étiquette morphologique dérivationnelle. L'abstraction y est toute relative, ce qui est typique des noms en -ness, dont le support figure souvent dans le contexte, de façon implicite ou explicite. La dérivation vers le nom y paraît donc incomplète, car elle s'inscrit sur la mémoire de la catégorie adjectivale. Les noms en -ness fonctionnent ici comme "prédicats déguisés en arguments » [Flaux, 1996: 87], dont la nature est essentiellement syncatégorématique, comme l'est spécifiquement celle des adjectifs et des verbes.

Pourquoi un tel « déguisement ", s'il s'agit de faire état d'une relation prédicative dite le plus directement dans la catégorie adjectivale? Au niveau syntaxique, ce déguisement permet au prédicat d'être thématisé, et de pouvoir entrer dans une relation directe avec d'autres arguments ou d'autres prédicats, ou des prépositions, comme c'est le cas dans les citations 2 et 3 . Les deux syntagmes nominaux de la citation 2 sont en effet introduits par la préposition upon, et définissent, circonscrivent ainsi un espace où le sujet devrait s'arrêter longuement (stop, dwell). Infiniteness désigne alors un lieu, dont l'extension est donnée par la prédication première, <sins/be infinite> ou <mercy/be infinites, et l'intension (l'infinité comme un lieu) par le sémantisme des verbes dynamiques et de la préposition de lieu. Dans la citation 3, comme dans $17 \%$ des noms en -ness de notre corpus, c'est une préposition de lieu qui introduit le syntagme nominal : in. Elle complète le verbe swallow, dont les connotations sémantiques "physiques " créent la rencontre entre concret et abstrait dans la proposition ends swallowed in infiniteness, que l'on peut tenter de formaliser :

\begin{tabular}{|l|l|l|}
\hline SN [abstrait] & Verbe [concret] & Prép SN [abstrait] \\
\hline
\end{tabular}

24 Alors que l'énoncé de la citation 1, proportion is no measure for infinity suit plutôt le schéma suivant:

\begin{tabular}{|l|l|l|}
\hline SN [abstrait] & Prédicat [abstrait] & Prép SN [abstrait] \\
\hline
\end{tabular} 
grande de l'énoncé, qui reste au niveau abstrait, une stabilité plus forte de la catégorie des objets abstraits, qui sont traités, syntagmatiquement, comme tels, c'est-à-dire entrant en relation avec des prédicats ou d'autres arguments dont les référents sont tout aussi abstraits. Lorsque le sujet parlant réalise une nominalisation en -ness, il fait autre chose qu'un simple emploi du lexique disponible; il est à la recherche d'une certaine expressivité (créativité lexicale) que ne permet pas -ity, et dont la motivation se perçoit grâce au choix d'une catégorie nominale gardant le souvenir et la trace linguistique de la catégorie adjectivale.

Il apparaît que les deux suffixés infinity et infiniteness n'ont pas le même mécanisme référentiel. Infiniteness ne fait référence que par une double médiation, la première par son noyau sémantique infinite, et la deuxième par rapport à ce à quoi infinite fait référence, c'est-à-dire, en première prédication, à sins dans le premier syntagme nominal de la citation 2. Infiniteness est donc syncatégorématique car prédicatif, tandis qu'il est difficile de dire d'infinity s'il est syncatégorématique ou catégorématique. En effet, la notion abstraite telle qu'elle est désignée par infinity dans la citation 1 réfère sans doute elle aussi par une médiation, en renvoyant au noyau sémantique adjectival infinite, mais cette médiation n'est pas obligatoire tant la notion nominale de l'infini s'est installée dans le lexique. Ce qui est sûr, c'est que la notion ne nécessite pas de référence supplémentaire à l'opération prédicative originelle pour être comprise. Elle a perdu toute spécificité d'une occurrence individuelle, elle n'est plus dans le particulier puisqu'elle n'a pas besoin de s'individuer pour faire sens. Bien sûr, c'est tout le problème de l'abstraction d'essayer de comprendre jusqu'où l'esprit humain est capable de donner du sens à l'infini en général sans avoir recours aux instances de l'infini (infinity peut-il faire référence de façon absolument autonome?), mais ce problème nous plonge dans des affres métaphysiques que l'on n'oserait regarder de trop près dans le cadre de cet article. Remarquons seulement que, si infinity fonctionne bien en catégorématique, c'est sans doute parce que les cas d'infini sont moins faciles à cerner que l'idée d'infini.

Cette différence entre les deux suffixés peut être expliquée en termes d'identité catégorielle ou de préconstruit de l'énoncé. En ceci, le nom en -ness est plus «concret » que le nom en -ity, si l'on accepte comme des critères du concret la matérialité (perceptibilité) du référent et son degré d'autonomie logico-référentielle à l'intérieur du syntagme. Dans le syntagme nominal the infiniteness of those sins, le premier argument $\mathrm{X}$ est sins, c'est par son biais que infiniteness continue de référer. Infinity vérifie donc moins qu'infiniteness le paradoxe du nom abstrait établi par Van Peteghem [1996 : 357] :

Le référent éventuel du nom abstrait ne peut être repéré que par l'intermédiaire de ce support concret. Dans ce sens, les abstraits se rapprochent du point de vue référentiel des verbes et des adjectifs, dont le référent, c'est-à-dire l'occurrence concrète, est également repéré à partir du sujet de la prédication.

«Sujet de la prédication » désigne ici sins, dont est prédiqué infinite. Mais ne faut-il pas remonter plus loin dans la genèse du syntagme the infiniteness of those sins? Cette occurrence concrète, Donne eût sans doute pu l'exprimer dans la catégorie verbale, car la nature syncatégorématique du nom en -ness est manifeste : le syntagme the infiniteness of those sins ne réfère en définitive pas si différemment du prédicat to sin infinitely. On constate en effet assez peu de différence, du point de vue référentiel, entre les trois énoncés suivants, où la relation d'attribution entre la qualité infinite et la notion sin est 
exprimée respectivement dans les catégories adjectivale, nominale et verbale [et adverbiale] :

1. the infiniteness of those sins

2. those sins are infinite

3. $\mathrm{we}^{7}$ have sinned infinitely

Le syntagme 1 thématise, par le nominal, la prédication, et le temps de l'énonciation ne coïncide pas avec le temps de la prédication; l'énoncé 2 fait coïncider temps de la prédication et temps de l'énonciation; l'énoncé 3 fait apparaître le sujet premier de la prédication, l'entité du monde extralinguistique à propos de laquelle la relation prédicative <sins/be infinite est vraie. Le mode verbal, relationnel, laisse place à l'expression d'un élément fondamental, et non apparent le plus souvent dans le nom en ness, le véritable sujet de la prédication.

Mais il faut encore ajouter un niveau d'interprétation : car si cette relation prédicative est vraie à propos d'un argument, elle est d'abord vérifiée, actualisée dans l'espace-temps de l'énonciateur, et implique, en définitive, le sujet parlant. Il nous faut en effet remonter au sujet même de l'énonciation si l'on veut retrouver l'occurrence « concrète » de la relation prédicative validée dans un espace-temps par un sujet parlant. Riddle [1985: 458] parle du trait « incarné » (embodied trait) que dénote le suffixe -ness, par différence avec le trait «abstrait» exprimé par le suffixe -ity. Dans le nom en -ness se manifeste l'origine énonciative, l'espace mental où la prédication <sins-be infinite> est vraie, c'est-à-dire, ici, l'esprit de John Donne. L'occurrence concrète du nom infiniteness serait le moment, actualisé dans l'espace-temps, où la prédication <sins-be infinite> s'est réalisée, vérifiée pour l'énonciateur; ce seraient les situations où les péchés de l'homme [dont la cloche sonne le trépas, et ceux de Donne lui-même], se sont avérés infinis, donc les situations énonciatives particulières définies par le double repère que constituent l'esprit de l'énonciateur et la relation prédicative (validée).

31 Dans le cas d'infinity, citation 1, on peut certes envisager de dire que Dieu est ce dont infinite est prédiqué, mais la prédication ne s'énonce pas aussi simplement que <God-be infinite> ; il pourrait tout aussi bien s'agir d'une définition de type God is infinity ou d'une sorte d'identification implicite avec relative nominale sujet what God is is infinity. Rien en tout cas dans le contexte proche ne permet de reconstruire une genèse prédicative certaine à cet énoncé générique.

Toutes les autres données de notre corpus vont dans ce sens : le nom en -ness émerge dans des contextes spécifiques, avec des déterminations à visée particularisante (article indéfini, $14 \%$ des occurrences, adjectif possessif, $15 \%$, démonstratif, $13 \%$, article défini non générique, $23 \%$, complément de préposition spécifiant le support de la prédication, $9 \%$ ), il entre dans des relations syntagmatiques avec des noms typiquement " concret ", à référent matériel, là où -ity est employé dans des contextes plus génériques, le plus souvent avec le déterminant $\varnothing$.

\section{Le haut degré}

\subsection{Affinité de -ness avec l'expression du haut degré}

Dans notre corpus, comme en anglais contemporain, le suffixe -ness s'ajoute le plus souvent à des qualités susceptibles de degré, comme dans dryness, loudness, idleness, fatness, 
weariness, etc. - dont les bases peuvent être modifiées par l'adverbe very. C'est une différence remarquable entre les suffixes -ness et -ity selon Baeskow [2012] : bien que les deux suffixes soient grammaticalement susceptibles de s'ajouter à des bases adjectivales gradables [sincereness/sincerity, sereneness/serenity], -ness semble préféré lorsque le contexte indique un haut degré de la propriété désignée, tandis que -ity nominalise des qualités indépendamment de leur structure scalaire. En cela encore, la nominalisation en -ness conserve un trait typiquement adjectival à la base suffixée. Fait encore plus remarquable, le nom en -ness, et ce même lorsqu'il dérive d'un adjectif non gradable, est souvent modifié par un syntagme adjectival indiquant le haut degré, comme dans les exemples suivants autour de aliveness et uniqueness, empruntés à Baeskow [2012 : 13] :

(2) From the start, these letters attest to Sarton's intense aliveness. (Sunday Times)

(3) We have no certain evidence that all of us have the potential for tumo, but there are strong reasons to believe that all of us can experience exceptional aliveness, and that such aliveness can be cultivated. (COCA)

(4) "The winners in each category achieved the highest 'uniqueness' index score in their particular categories." (Sunday Times)

(5) But is this broad and almost pantheistic frame of reference in itself any more valid than, for example, the traditional Christian affirmation of the absolute uniqueness of Jesus as the incarnation of God? (BNC)

Dans ces exemples, -ness montre sa capacité à ajouter le trait (haut degré) à des bases non gradables; cette valeur ajoutée résulte de la nominalisation, dont l'interprétation doit être phénoménologique, comme je l'explique plus bas. L'affinité sémantique entre -ness et le haut degré est visible dans un autre fait mentionné par Baeskow et récurrent dans notre corpus : il est fréquent que -ness s'ajoute à une base déjà suffixée dont le suffixe signifie le haut degré, comme c'est le cas des suffixes -ous et -ful. Ces cas (suffixation de ness sur base suffixée en -ous et -ful) représentent $20 \%$ de nos occurrences (e.g. ingloriousness, fruitfulness $\left.{ }^{8}\right)$. Or -ous, suffixe issu du Latin classique, signifie toujours une forme d'abondance, comme indiqué dans la définition de l'OED : "forming adjectives with the sense 'abounding in, full of, characterized by"' - sens bien sûr également véhiculé par le suffixe -ful. Dans notre texte, la création de noms en -ness à partir de ces deux suffixes ( licentiousness, graciousness, thankfulness, watchfulness, righteousness, etc.) expriment à chaque fois, de la part de John Donne, une réaction forte à un phénomène perçu à un haut degré et ancré dans une situation particulière. Cette situation énonciative, caractérisée par l'implication forte de l'énonciateur, est la situation motivant prototypiquement les métaphores : elle suscite un besoin de créativité lexicale adéquat.

\subsection{Righteousness}

35 Étudions brièvement le " parcours » syntagmatique du nom righteousness dans ce passage de l'Expostulation 5 :

Righteousness is immortal; I know thy wisdom hath said so; but no man, though covered with the righteousness of thy Son, is immortal so as not to die; for he who was righteousness itself did die. I know that the Son of Righteousness, thy Son, refused not, nay affected, solitariness, loneness, many, many times [Exp. 5: 2554].

Ici, Donne reprend une citation biblique, dans laquelle le nom abstrait righteousness est traité syntaxiquement dans un contexte de type générique, qualification universelle, de 
forme prédicative simple, A is b. C'est le point de départ (0) d'un trajet suivant les 3 étapes suivantes :

\begin{tabular}{|l|l|}
\hline 0. & righteousness \\
\hline 1. & covered with the righteousness of thy Son \\
\hline 2. & he who was righteousness itself \\
\hline 3. & the Son of Righteousness \\
\hline
\end{tabular}

1. Righteousness est d'abord inclus dans un syntagme prépositionnel introduit par with et complément d'un participe passé adjectivé aux connotations physiques, covered, qui implique l'actualisation du procès [cover]; au même moment, tandis que dans la citation biblique la notion abstraite, autonome référentiellement, ne signale aucune trace d'une genèse prédicative quelconque, le nom righteousness est à présent complété par l'argument dont righteouness est prédiqué, le nom «support » du terme de base du nom en -ness - dans la terminologie de la psychomécanique du langage qu'on a adoptée ici thy Son.

On a donc, dans un premier temps, réattribution de la qualité suffixée en -ness righteous à l'argument support thy Son sans quitter une forme nominale sémantiquement liée à une action réalisée et concrète covered.

2. Dans une seconde formulation, righteousness est attribué entièrement au sujet he coréférent à thy Son, dans la proposition relative: (he) who was righteousness itself. Il y a alors identification totale entre la notion righteousness et la personne du fils de Dieu, le réfléchi itself soulignant l'adéquation parfaite de cette incarnation de la notion. L'incarnation de cette qualité ne vaut jamais autant que dans le corps du Christ; c'est donc que le nom en -ness est actualisé dans l'espace-temps, où il accomplit sa vocation sémantique à désigner une forme absolue et incarnée. Ce qui permet au nom righteousness d'être repris une troisième fois dans le syntagme nominal en of, the Son of Righteousness.

3. Il paraît délicat de déterminer la relation sémantique entre le nom tête [head-h] de ce syntagme, the Son, et son complément de nom [determiner-d] Righteousness. A première vue, et si l'on respecte la cohérence discursive - thy Son venant d'être identifié comme réalisant le mieux righteousness, le syntagme $h$ of $d$ signifierait en genèse $h$ is $d$, qui rejoindrait du point de vue du sens les structures formalisées par Huddleston et Pullum [2002: 477] comme suit: « h has human property of $d$, ou $h$ is supreme example of $d$ ", avec les exemples respectifs the men of honour et the battle of all battles.

Mais l'utilisation de la majuscule incline à croire que Righteousness fonctionne comme un nom propre, ce qui, si h of $\mathrm{d}$ signifie $\mathrm{h}$ is $\mathrm{d}$, ferait de cette structure de complémentation un syntagme sémantiquement redondant; et l'on sait que, dans une duplication magnanime, le nom du fils de Dieu est aussi le nom de Dieu, et le syntagme thy Son apposé au syntagme the Son of Righteousness appuie cette interprétation plus commune, avec une complémentation indiquant classiquement un signe d'appartenance. $\mathrm{H}$ is $\mathrm{d}$ révèlerait sémantiquement $\mathrm{d}$ has $\mathrm{h}$, et serait l'autre forme d'une structure génitive possible Righteousness's Son, où le nom propre identifierait bien le père du fils, et serait un nom de Dieu. De plus, dans une construction en of, la focalisation se fait à gauche, comme le 
souligne Cotte [1984: 109], qui met en valeur l'effet «actualisant» de of : «qu'il y ait extraction ou modification, of marque un passage au réel, qui implique une rupture entre le plan de la notion et de l'actualisation ». Dans the Son of Righteousness, c'est bien le fils qui incarne, actualise la droiture du père, et la notion righteousness, dont la remotivation sémantique a déjà été suffisamment thématisée, est si bien établie, ancrée en la personne divine [du fils au père], que son extensité est à présent minimale, son intensité maximale, et fonctionne comme le nom de Dieu. Le nom en -ness est ainsi devenu nom propre, au plus loin de la référence universelle. Dans les termes de la psychomécanique du langage [Lowe 2007 : 253], le nom propre désigne l'extensité, champ de désignation en discours, la plus étroite possible :

C'est en raison de l'extrême densité de son contenu idéogénique, lequel atteint au maximum concevable de la particularisation, que le type de mot auquel correspond le nom propre n'offre aucune possibilité de généralisation et ne peut en conséquence faire concept.

Le nom righteousness indique bien ici le plus haut degré, incarné dans la personne du fils de Dieu. Comme dans la plupart de nos données, il n'y a pas eu mention préalable de la base adjectivale seule, et le nom en -ness ne doit pas être compris dans une optique de cohérence discursive (avec une dimension anaphorique) mais en termes de contexte énonciatif privilégié.

\section{Métaphore grammaticale}

41 La nominalisation en -ness constitue en réalité un cas de métaphore grammaticale ${ }^{9}$. Comme tous les énoncés métaphoriques, dont la métaphore lexicale, très étudiée, elle révèle un transfert de traits symboliques d'une catégorie à l'autre, ici de la catégorie nominale (stabilité et matérialité du référent signifiant la perceptibilité à un haut degré) à la catégorie adjectivale. La signification symbolique des parties du discours permet de créer un sens ajouté lorsque celles-ci sont momentanément (métaphores émergentes) dissociées de leurs référents types. La grammaire serait cognitive en cela qu'elle véhicule des concepts et des symboles qui participent à la construction du sens :

Grammatical structures do not constitute an autonomous formal system or level of representation: they are claimed instead to be inherently symbolic, providing for the structuring and conventional symbolization of conceptual content. Lexicon, morphology and syntax form a continuum of symbolic units, divided only arbitrarily into separate components [Langacker 1990: 1].

La métaphore, qu'elle soit lexicale (Juliet is the sun) ou grammaticale, apporte le trait [matériel-concret] à des entités abstraites, ce qui permet de restituer aux sentiments leur « entièreté » phénoménologique [Lowe 2007]. Les catégories de l'abstrait et du concret sont un support essentiel à ce transfert.

La spécificité de la dérivation en -ness (résultant de sa "transparence ») réside dans sa capacité à faire percevoir cette décatégorisation émergente, et par là-même à donner accès à la motivation du sujet parlant.

\subsection{Motivation métaphorique}

La même motivation préside à toutes les métaphore : c'est une motivation cognitive qui répond à un besoin spécifique - s'approprier le langage pour exprimer l'intensité d'une expérience, qui semble unique au sujet parlant. Ainsi les sentiments forts, qui impliquent 
l'énonciateur, donnent les situations énonciatives les plus propices à la création de métaphores. Une métaphore prototypique implique le sujet parlant, et mène à l'implicitation de sa présence dans le discours ${ }^{10}$. Une interprétation phénoménologique de la métaphore est nécessaire : impressionné par un phénomène qu'il perçoit à un haut degré, le sujet parlant éprouve le besoin de déranger le langage et de laisser son empreinte, créative, dans le discours. On peut parler de "lyrisme indirect», où « indirect» caractérise la non-lexicalisation de l'expression des sentiments. Par la nominalisation en -ness, le sujet parlant exprime un sentiment/affect d'une façon discrète, invisible à la surface du discours. Cette création lexicale a vocation à être unique et se produit en réaction à une perception de carence lexicale. Motivation de la créativité, cette perception est justifiée ou non, et peut être plus imaginaire que réelle, comme le souligne Bauer [2001: 41] : « a real or perceived gap in the speaker's lexicon ».

Il est fréquent que la motivation métaphorique soit perceptible dans plusieurs faits de langue concomitants ; le nom en -ness est ainsi parfois associé dans notre corpus au there dit «existentiel», qu'il est possible [Groussier 1996: 156] de comprendre comme un emploi métaphorique de l'adverbe de lieu, celui-ci représentant la situation énonciative comme un lieu :

1. there is a propenseness to diseases in the body [Med. 22: 134]

2. there is [...] no inextricableness in thee (my God) [Exp. 6: 29]

3. there rises a kind of phoenix out of the ashes, a fruitfulness out of that

which was barren before [Med. 22: 135]

Les noms en -ness sont des noms ad hoc. Dans ces trois exemples, leur référent est doublement situé, une première fois par there, une deuxième fois par les syntagmes prépositionnels en fin d'énoncé; en 1 et 3 , ce référent semble saisi au moment où il se manifeste, se particularise pour l'énonciateur, comme l'indique l'emploi de l'indéfini A. En 3., on pénètre, grâce aux deux métaphores grammaticales, dans l'espace intime (conscience du sujet parlant) où l'éclosion de cette fertilité est observée. Il y a manifestation de la qualité fruitful dans la conscience de l'énonciateur, qui s'en saisit, s'y arrête, et la fait exister comme une substance. Donne constate l'émergence d'une fertilité à partir d'un terrain stérile, la qualité fruitful est perçue au moment où elle émane, elle est une apparition qui le réjouit et promet la délivrance. Le lieu physique est mentionné en fin d'énoncé that which was barren before, de même que le complément circonstanciel de lieu in the body reformule le sujet de la prédication <body/be propense to diseases>, sujet réel déplacé en fin d'énoncé et remplacé par le sujet grammatical adverbe de lieu there. À chaque fois, les énoncés circonscrivent un espace énonciatif fort, que le référent du nom en -ness occupe exclusivement, à un haut degré.

\subsection{Le sens de la catégorie nominale}

En nominalisant, le sujet parlant attribue le sens symbolique de la catégorie nominale à la qualité prédiquée. Langacker [1990: 75] caractérise ainsi la prédication "nominale» comme délimitant une « région » constituée d'entités reliées entre elles, et la prédication «relationnelle»[verbes et adjectifs] comme établissant des liens entre des entités séparées :

A nominal predication presupposes the interconnections among a set of conceived entities, and profiles the region thus established. On the other hand, a relational 
predication presupposes a set of entities, and profiles the interconnections among these entities. devenue l'absolu momentané du sujet parlant. C'est pourquoi -ness ajoute le trait [haut degré] même lorsque sa base n'est pas gradable. Il a été montré que la catégorie du nom avait une signification symbolique stable, plus stable que les autres catégories de discours, et que la nominalisation induisait ainsi certaines connotations de type [concretperceptibilité] :

The concept-forming power of words has its strongest effects with nouns. The most prototypical examples of nouns, namely nouns denoting classes of concrete entities, refer to things. As a consequence, they lend themselves much more readily to a conceptualization of what they stand for as 'things' and this greatly encourages the illusion of reification. But even nouns denoting abstract entities, relations or properties are affected by this illusion. [Schmid $2000: 17]$

\subsection{Créativité à usage unique}

Le nom en -ness prototypique est celui qui parvient à transmettre cette valeur " concrétisante" de la façon la plus perceptible, car il renvoie alors à la motivation énonciative en jeu - le besoin de créativité lexicale. Dès qu'il sort du cadre unique où il a été créé, dès qu'il cesse d'être un nonce-word, il perd de sa métaphoricité, de sa perceptibilité. Les notions de gradient et de prototypicité permettent de penser les variations du processus métaphorique à partir du moment où la métaphore entre dans le discours. La lexicalisation est le point d'aboutissement de ce processus évolutif, signalant la [dé]gradation de la motivation métaphorique : lorsque le nom en -ness entre dans le dictionnaire, il cesse de référer vers un acte linguistique créatif.

Pourtant, les locuteurs - et ils sont majoritaires - qui n'en ont pas conscience / connaissance, continuent, avec bonheur, de réagir à un manque lexical ressenti par ce qu'ils perçoivent comme une forme, fût-elle discrète et "quotidienne", de créativité lexicale.

\section{Conclusion : arguments contre la TMC (Théorie de la Métaphore Conceptuelle)}

50 S'il est juste de dire avec les tenants de la Theory of Conceptual Metaphor [Lakoff 1987, Johnson 1987] que la métaphore est quotidienne et de nature cognitive, il est faux de l'interpréter comme une projection intellectuelle de domaines et de la situer non pas au niveau du langage mais au niveau de la pensée ${ }^{11}$. La métaphore est affaire de langage car elle exploite les catégories, lexicales ou grammaticales, et crée une saillance au niveau syntagmatique. Elle ne se situe pas avant le langage, elle ne lui préexiste pas, car elle se réalise en lui. Elle exploite les codes sémantiques et les attentes générées par les catégories grammaticales. La métaphore n'est donc pas d'abord affaire de concept ${ }^{12}$. Les métaphores ne deviennent structurelles, communes à tous, que lorsqu'elles ont connu une évolution lexicalisante et perdu la trace de la motivation métaphorique type, qui fait retour vers l'unicité créative du sujet parlant. Certes, parce qu'elles correspondent à une expérience cognitive souvent universelle, elles sont aisément remotivables et peuvent "ressusciter", rejouant à chaque fois le besoin du sujet parlant de faire preuve linguistique de créativité lexicale. Mais les métaphores traitées par la TCM ne sont que la 
trace enregistrée a posteriori, dans le lexique [lexique alors disponible pour structurer l'esprit de futurs locuteurs], d'un processus moins actif ou expressif. L'expressivité métaphorique est susceptible de (dé)gradation ; elle s'use aussi vite que se fixe le langage.

\section{BIBLIOGRAPHIE}

\section{Corpus}

DONNE John, Devotions upon Emergent Occasions, SPARROW John [éd.], Cambridge: The University Press, 1624, 1923.

\section{Sources linguistiques et dictionnaires}

BAUER Laurie, Morphological Productivity, Cambridge : Cambridge University Press, 2001.

BAUER Laurie, English Word-Formation, Cambridge: Cambridge University Press, 1983.

BAESKOW Heike, “-ness and -ity: Phonological Exponents of $\mathrm{n}$ or Meaningful Nominalizers of Different Adjectival Domains?", Journal of English Linguistics, 2012, 40: 6.

COTTE Pierre, « OF et la modification », Sigma, 7, $1984:$ 75-93.

CROFT William \& CRUSE Alan D. [éds.], Cognitive Linguistics, London: Cambridge University Press, 2004.

CUYCKENS Hubert, DIRVEN René \& TAYLOR John R. (éd.), Cognitive Approaches to Lexical Semantics, Berlin: Mouton de Gruyters, 2003.

GOES Jan, L'adjectif : entre nom et verbe, Bruxelles, Paris : Duculot, 1999.

GROUSSIER Marie-Line \& RIVIÈRE Claude, Les mots de la linguistique : lexique de linguistique énonciative, Gap, Paris : Ophrys, 1996.

ELLRODT Robert, L'inspiration personnelle et l'esprit du temps chez les Poètes Métaphysiques anglais, 3 vol. , Paris, J. Corti, 1960.

HALLIDAY Michael A.K, révisé par MATTHIESSEN Christian M.I.M., An Introduction to Functional Grammar, 3rd éd., London: Arnold, New York: Oxford University Press, 1985, 2004.

HUDDLESTON Rodney \& PULLUM Geoffrey K., The Cambridge Grammar of the English Language, Cambridge: Cambridge University Press, 2002.

JOHNSON Mark, The Body in the Mind : the Bodily Basis of Meaning, Imagination and Reason, Chicago: University of Chicago Press, 1987.

LOWE Ronald, Introduction à la psychomécanique du langage, vol. I., Québec : Presses de l'Université de Laval, 2007. 
LANGACKER Ronald W., Investigations in Cognitive Grammar, Berlin: Mouton de Gruyter, 2009.

LANGACKER Ronald W., Cognitive Grammar : A Basic Introduction, Oxford: Oxford University Press, 2008.

LANGACKER Ronald W., Concept, Image, and Symbol : the Cognitive Basis of Grammar, Berlin-New-York: Mouton de Gruyter, 1990.

LAKOFF George, Women, Fire, and Dangerous Things, Chicago: The University of Chicago Press, 1987.

MARCHAND Hans, The Categories and Types of Present-Day English Word-Formation : a Synchronic-

Diachronic approach, Wiesbaden: O. Harrassowitz, 1960.

NEVEUX Julie, « It girl, it bag et le pronom it : le sens du pronom neutre non anaphorique ",

Anglophonia/Sigma 32, $2012:$ 63-84.

NEVEUX Julie, "La métaphore 'inappropriée' : échec du partage sentimental et flou résultatif », Lexis Spécial 2, 2010, disponible à

http://lexis.univ-lyon3.fr/spip.php?article155

Oxford English Dictionary, 2009, Version électronique, Copyright @ Oxford University Press, disponible sur http://dictionary.oed.com/

PANTHER Klaus-Uwe, THORnBurg Linda L. \& BARCELona Antonio (éd.), Metonymy and Metaphor in Grammar, Hamburg, Córdoba : John Publishing Company, 2009.

RIDDLE Elizabeth M., "A historical perspective on the productivity of the suffixes -ness and -ity", in FISIAK Jacek [ed.], Historical semantics historical word-formation, Berlin: Mouton de Gruyter, 1985: 435-461.

schmid Hans-Jörg, English Abstract Nouns as Conceptual Shells: From Corpus to Cognition, Berlin, New York: Mouton de Gruyter, 2000.

WILliams Theodore, “On the -ness Peril”, American Speech, vol. 40, No. 4, 1965: 279-286.

VAN PETEGHEM Marleen, « Attributs nominaux à substantif noyau abstrait », in FLAUX N., GLATIGNY M. \& SAMAIN D. [éds.], Les noms abstraits : histoire et théories, Villeneuve-d'Ascq : Presses universitaires du Septentrion, $1996:$ 357-366.

ZAJICEK Jacques, Notions essentielles d'anglais [éléments de stylistique], Paris : Sirey, 1965.

\section{NOTES}

1. Description détaillée de cette donnée du CoCA : 2007, Chef on a Shoestring; Mizuna executive chef Frank Bonanno makes a three-course meal using fresh food from farmers market, Source, CBS_SatEarly.

2. Corpus choisi en raison du grand nombre de métaphores grammaticales (et lexicales) qu'il contient : l'intérêt du poète métaphysique pour l'expression linguistique des entités non perceptibles alimente une écriture hautement métaphorique, alliant sans cesse l'abstrait et le concret. Pour Ellrodt [1960:256], Donne use du sensible pour « faire surgir en l'esprit ce que l'on pourrait nommer concept concret ».

3. Bauer [2001: 157] situe cette prise de conscience un peu plus tard, dans les années 1980 : "words with the suffix -ness are ones of which the general public was made aware during the 80s and early 90 s". 
4. Dont voici la liste: ableness affectionateness alertedness aliveness aloneness ambitiousness amiableness animalness apartness archaicness belongingness betterness braveness briefness carness cleanness comfortableness competitiveness contentness coquettishness creativeness crudeness cruelness cunningness dayness decorativeness descriptiveness desolateness detectiveness differentness divineness electness equalness erraticness evilness falseness familiarness ferociousness flowingness franticness frigidness futileness givenness givingness good-naturedness greediness hereness highness (height) hoitytoityness horridness hospitableness humanness humbleness impatientness impetuousness impoverishness inferiorness initiativeness inner-directiveness inness insecureness justness (justice) jealousness lostness maleness maliciousness matureness messiness miserableness moistness morbidness muchness naiveness nobleness nothingness nounness obtuseness oral hungriness passionateness passiveness peanutiness piousness pointedness preciseness properness proudness pureness puritanness realness regularness relaxedness responsiveness (responsibility) rightness rigidness routineness sameness saneness scaredness scientificalness sensualness shamefulness shortness (brevity) showeryness simpleness slovenness socialness selectness solidness sorriness stereotypeness stomach hungriness subdueness subtleness successfulness superficialness superiorness tenseness (tension) terribleness thereness thingness timidness togetherness tragicness unbreakableness uncleanness unguardedness universalness unpeacefulness untrueness vainness verbness vitalness virtuousness (virtuosity!) wellness wellroundedness wiseness (wisdom) worthwhileness.

5. [I, 319, publié par Cross en 1885].

6. Pour se référer aux Devotions (édition Sparrow de 1923), on utilise les abréviations suivantes : Exp. pour Expostulation, Pr. pour Prayer, Med. Pour Meditation.

7. On choisit le pronom «we », car Donne fait ici référence à l'homme dont la cloche signale le trépas, et s'inclut dans les pécheurs quémandant le pardon divin.

8. Les noms en -ity ne s'attachent jamais à ce suffixe; ils sont le plus souvent suffixés à une base simple (environ $80 \%$ des occurrences); le suffixe -al précède -ity dans $11 \%$ des occurrences.

9. Halliday $[1985,2004]$ considère également la nominalisation comme un cas de métaphore grammaticale, qu'il appelle "idéationnelle », et qui consiste à traiter (downgrade) comme une entité une qualité ou un procès. Mais il s'intéresse surtout à ces métaphores dans le cadre d'une cohérence discursive, et dans les domaines administratifs, légaux et scientifiques ; il reconnaît cependant, comme trait systémique effectif du processus de métaphorisation, un glissement du mode logique au mode expérientiel, an experientialization of experience [2004:641].

10. Voir Neveux [2012, 2010]. Halliday [2004:641] interprète cette implicitation comme une "perte » de contenu informationnel (par rapport à un énoncé typique, dit «congruent ») : "the perceiver is left implicit."

11. "In short, the locus of metaphor is not in language at all, but in the way we conceptualize one mental domain in terms of another. The general theory of metaphor is given by characterizing such cross-domain mappings. And in the process, everyday abstract concepts like time, states, change, causation, and purpose also turn out to be metaphorical. The result is that metaphor [that is, cross-domain mapping] is absolutely central to ordinary natural language semantics, and that the study of literary metaphor is an extension of the study of everyday metaphor". Ou encore: "Metaphorical language is a surface manifestation of conceptual metaphor". [En ligne] George Lakoff, 1992, [consulté le 25 mars 2013], disponible sur http://www.ac.wwu.edu/ market/ semiotic/lkof_met.html

12. La métaphore existe bien sûr dans d'autres domaines en-dehors de la langue, musique, peinture, cinéma, mais à chaque fois c'est en bousculant les modes d'expression propres à ce médium spécifique qu'elle parvient à rendre perceptible sa motivation, sa raison d'être [faire exister un qualia esthétique]. 


\section{RÉSUMÉS}

Cet article interprète la nominalisation en -ness comme un cas de métaphore grammaticale, dans l'optique d'approches cognitives récentes [Langacker 2009, Panther et alii 2009]. Le suffixe -ness est l'un des plus productifs de la langue anglaise, parce qu'il est «transparent »: il permet à la base prédicative de continuer à s'exprimer totalement à l'intérieur du nom dérivé. Le processus de décatégorisation est ainsi perceptible, et donne accès à la créativité lexicale du sujet parlant. Les noms en -ness sont prototypiquement des noms à usage unique, créés par l'énonciateur lors d'une situation dont il veut exprimer l'unicité. Comme dans les métaphores grammaticales, le transfert de traits symboliques (ici de la catégorie du nom à celle de l'adjectif) mène à une forme de concrétisation. La Théorie de la Métaphore Conceptuelle ne rend pas compte de ce fait : toutes les métaphores sont motivées par la recherche d'une différence linguistique, qui diminue au fur et à mesure que les métaphores se lexicalisent et se mettent à constituer des domaines structurés et « conceptuels». Les métaphores prototypiques ne constituent pas des projections cognitives préétablies. Métaphoricité et lexicalisation sont en réalité des processus évolutifs contraires.

In this paper, -ness nominalization is interpreted as a case of grammatical metaphor, following recent cognitive approaches such as Langacker [2009], Panther et alii [2009]. -ness suffix is one of the most productive suffixes in the English language, because it is "transparent": it allows the predicative stem to remain fully perceptible within the derived noun. The decategorization process is thus perceptible, giving access to the speaker's lexical creativity. -ness words are prototypically nonce-words, created by the speaker in a situation whose unique dimension (s)he needs to express; as in lexical metaphors, the transfer of symbolic traits (from the nominal category to the adjective one) leads to a form of concretization. Conceptual Metaphor Theory fails to take this fact into account: all metaphors are motivated by the search of a linguistic difference, which diminishes as metaphors get lexicalized and turn into structural, "conceptual" mapping. Prototypical metaphors are not pre-established cognitive projections. Metaphoricity and lexicalization are opposite evolutive processes.

\section{INDEX}

Mots-clés : suffixe -ness, décatégorisation, lexicalisation, créativité, métaphore grammaticale Keywords : suffix -ness, decategorization, lexicalisation, creativity, grammatical metaphor

\section{AUTEUR}

JULIE NEVEUX

Université Paris 4 - Sorbonne

jneveux@hotmail.fr 